\title{
Hydatid cyst of the lung in pregnancy: A rare case presentation
}

\author{
Ahmad Shirinzadeh ${ }^{1}$, Amene Hadadan², Sedighe Vaziribozorg ${ }^{3}$ \\ 1 Assistant Professor, Department of Surgery, Shahid Sadoughi University of Medical Sciences, Yazd, Iran \\ 2 Assistant Professor, Department of Gynecology, Shahid Sadoughi University of Medical Sciences, Yazd, Iran \\ 3 Researcher, MD, Department of Otolaryngology-Head and Neck Surgery, Otorhinolaryngology Research Center, Shahid Sadoughi \\ University of Medical Sciences, Yazd, Iran \\ *Corresponding Author: Dr. Amene Hadadan, Assistant Professor, Department of Gynecology, Shahid Sadoughi University of \\ Medical Sciences, Yazd, Iran. \\ Email: vaziribozorg.s@gmail.com; Tel: +9835381133372
}

Received: February 25, 2020; Accepted: March 31, 2020

\begin{abstract}
Hydatid cyst of the lung has been seen in $30 \%$ of the cases of hydatidosis in some series. In our case presentation, a 29 -year old female, gravida 1 , referred to the hospital with acute and severe pain in the lower left hemithorax, while she was in the 26th week of pregnancy. The pain was pleuritic and was accompanied with tachypnea. The patient was hospitalized with suspected pulmonary embolism. The chest X-ray and computed tomography showed a cystic mass in the lower left lung. After a diagnosis of hydatid cyst, the patient underwent surgery and all of her symptoms were resolved. As Albendazole is a medication from pregnancy category $\mathrm{C}$, and since in our case, the patient underwent surgery and all of her symptoms were resolved, we can conclude that surgery is a safe and appropriate treatment for Hydatid cyst of the lung in pregnancy.
\end{abstract}

Keywords: Hydatid cyst, Lung, Pregnancy.

\section{INTRODUCTION}

Hydatid cyst is a disease transmitted from animals and infects humans as an intermediate host. By consuming the water, vegetables, and fruits infected with the parasite eggs, the larval stage begins in the intermediate host and leads to hydatid cysts of the lung and the liver [1]. Lung involvement, which follows hepatic infestation in frequency, has been seen in 30\% of the cases of hydatidosis in some series [2]. Hydatid disease in pregnancy is a rare condition, with an incidence of $1 / 20,000$ to $1 / 30,000$ deliveries [3]. The clinical features of lung hydatid cysts, if not ruptured, depend on the site and size of the cyst [4]. Cysts that have grown in the lung environment can cause bronchial compression (a dry cough) or chest pain (feeling acute pressure in the chest wall). Chest X-ray and CT scans are the common ways to diagnose the hydatid cysts. In radiography, the cyst appears as a spherical homogeneous mass with distinct edges [5]. Ultrasonography and echocardiography should also be performed to evaluate the concomitant liver and heart hydatid cyst. Surgery is the main treatment for lung hydatid cyst [6].

\section{CASE REPORT}

The patient was a 29-year old female (26 weeks pregnant) who referred to the women's ward. The patient had a complaint about severe pain in the left part of her chest that began suddenly two days earlier. The patient's had a pleuritic pain and it was accompanied by nausea and anorexia. The patient had uninvestigated dry coughs since the beginning of her pregnancy. Two days prior to her referral to our center, she was hospitalized in another clinic with a diagnosis of pneumonia (without CXR) and IV antibiotics were prescribed for her. Because of no improvement in symptoms, the patient left that center and came to our hospital. As a result of acute severe chest pain, dyspnea and tachypnea, she admitted with the first diagnosis of pulmonary embolism. The patient's blood pressure was 110/70 $\mathrm{mmHg}$, pulse rate was $90 / \mathrm{min}$, body temperature was $36^{\circ} \mathrm{C}$, respiration rate was 22 / $\mathrm{min}$, and oxygen saturation was $96 \%$. In the examination of her chest, we found that the lower regions of the left lung had dullness and the respiratory sounds decreased in these parts so that no rhonchi or crepitus was heard. In the examination of her abdomen, uterus size showed that the patient was at the 26th week of pregnancy. The heart and nervous examination did not show any abnormal point. The following results were achieved from the laboratory tests: White blood cell: 10.600 , neutrophil 85 percent, eosinophil 2.5 percent, hemoglobin $11.1 \mathrm{gr}$ and platelet 328000 . The arterial blood gas $(A B G)$ test showed respiratory alkalosis and electrocardiogram (ECG) showed normal sinus rhythm. The CXR revealed a 
circular and large lesion of size $11 \times 12 \mathrm{~cm}$, which involved the lower lobe of the left lung (fig.1:A). At this stage, after observing the mentioned lesion, the possibility of pulmonary embolism was removed from our diagnosis. For further examination of the lesion, we requested thoracic CT without contrast. The CT scan showed a cystic lesion of $125 \times 113 \times 77 \mathrm{~mm}$ in the lower lobe of the left lung with a thick wall, which was suggestive of hydatid cyst or pneumonia (fig.1:B). After a diagnosis of the hydatid cyst, abdominal and pelvic ultrasonography was also performed, but no cystic lesions were observed. Hydatid cyst antibodies were requested, the immunoglobin $\mathrm{G}$ ( $\mathrm{lgG}$ ) of hydatid cyst was $631 \mathrm{mg}$ / dl and its immunoglobin M (IgM) was 108mg / dl. Patient's echocardiography was normal. Ultimately, the lung embolism was rejected and the hydatid cyst was diagnosed. Regarding the patient's chronic cough, her lack of improvement in the chest pain, the gastrointestinal symptoms noted by the patient, and the large size of the pulmonary cyst, we decided to select surgery for the patient's treatment. Posterolateral thoracotomy was performed (fig.1:C) and a hydatid cyst with a size of $14 \times 13 \mathrm{~cm}$ was removed from the lingual lobe (as opposed to the report of CXR and CT, which located the cyst in the left lower lobe) (fig.1:D). The lower lobe of the left lung was completely collapsed due to bronchial obstruction caused by the mass pressure, which was completely opened immediately after cyst removal (fig.1: E, F).

\section{DISCUSSION}

Hydatid cyst in adults is more common in the liver and lungs [7]. In most patients, the cough is a common symptom. The two causes of coughing in these patients include the cyst's rupture into the airways and the stimulatory effect of the growing cyst on the airways (such as our patient). In the studied patient, dry coughs aggravated as the pregnancy progressed and finally the patient referred to the hospital with severe and acute chest pain. This acute and severe pulmonary pain in the chest was caused by cystic pressure on the chest wall, which improved after the surgery. Treatment of hydatid disease during pregnancy is still debatable because the rupture risk may lead to anaphylaxis shock or delay in the fetal growth in preterm labor and the fact that no definitive guideline is available in this case. Albendazole is a medication from pregnancy category $\mathrm{C}$, which is prescribed only in the case that its benefits are greater than its harms during pregnancy. The physician may start this medicine during pregnancy, especially if the critical period of organogenesis of the fetus is completed [8].
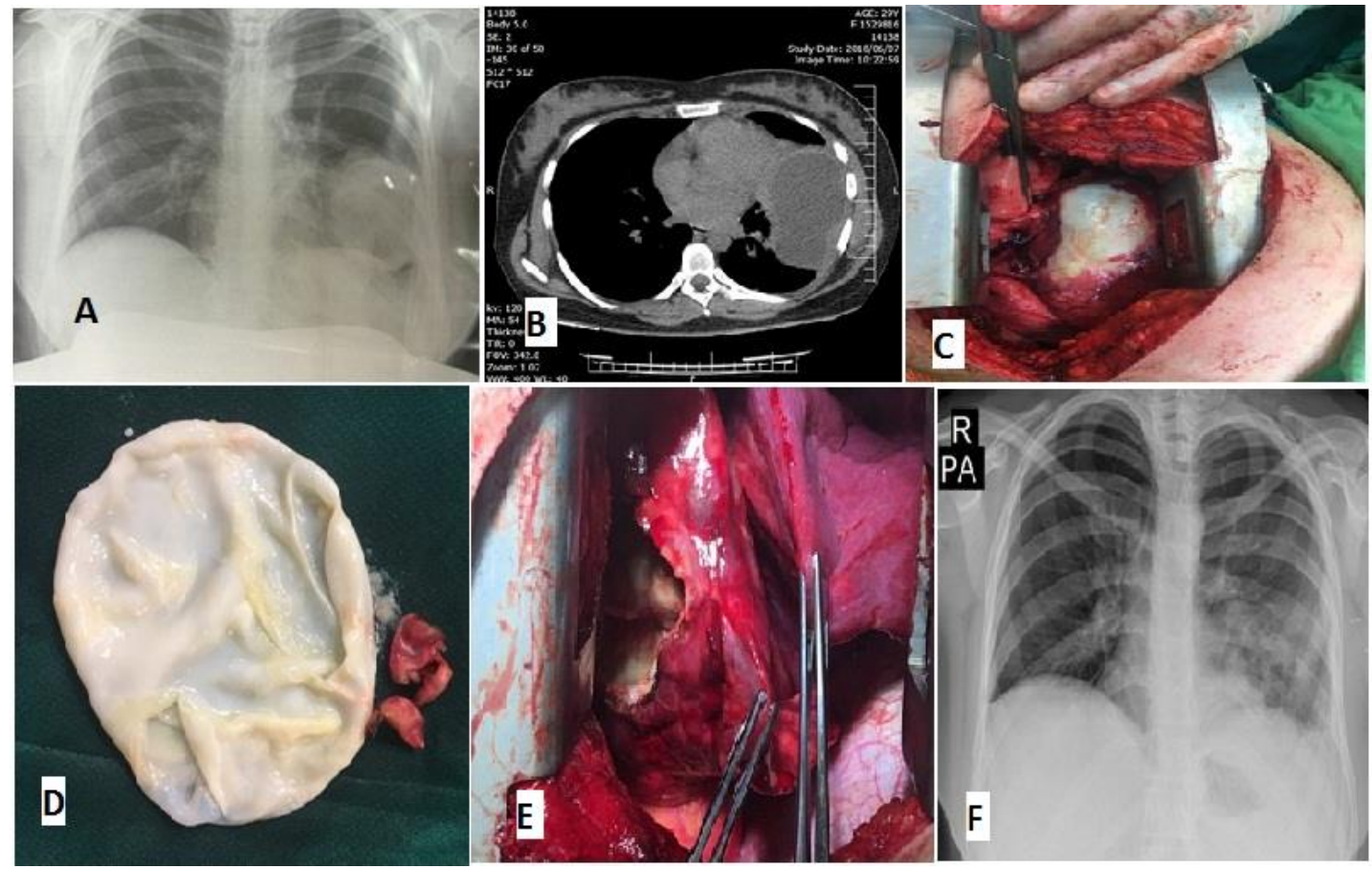

Fig.1: A: The CXR revealed a circular and large lesion of size $11 \times 12 \mathrm{~cm}$, which involved the lower lobe of the left lung. B: The CT scan showed a cystic lesion of $125 \times 113 \times 77 \mathrm{~mm}$ in the lower lobe of the left lung with a thick wall, which was suggestive of hydatid cyst or pneumonia. C: Posterolateral thoracotomy was performed and a hydatid cyst with a size of $14 \times 13 \mathrm{~cm}$ was removed from the lingual lobe. D: Removed cyst. E: After removal of the cyst. F: The CXR revealed the mass removal.

\section{CONCLUSION}

As Albendazole is a medication from pregnancy category $\mathrm{C}$, and since in our case, the patient underwent surgery and all of her symptoms were resolved, we can conclude that surgery is a safe and appropriate treatment for Hydatid cyst of the lung in pregnancy.

\section{Acknowledgement}

* Study protocol was in accordance with the latest Declaration of Helsinki for medical research involving human subjects and was approved by the local ethics committee.

*This article does not contain any studies with animals performed by any of the authors. 


\section{Conflict of Interest}

We declare that we have no conflict of interest.

\section{Financial Support}

None declared.

\section{REFERENCES}

1. Mandal S, Mandal MD. Human cystic echinococcosis: epidemiologic, zoonotic, clinical, diagnostic and therapeutic aspects. Asian Pacific journal of tropical medicine. 2012;5(4):25360.

2. Ramos G, Antonio Orduña M, Mariano García-yuste M. Hydatid cyst of the lung: diagnosis and treatment. World journal of surgery. 2001;25(1):46-57.

3. Hijazi MH, Al-Ansari MA. Pulmonary hydatid cyst in a pregnant patient causing acute respiratory failure. Annals of thoracic medicine. 2007;2(2):66.

4. Shehatha J, Alizzi A, Alward M, Konstantinov I. Thoracic hydatid disease; a review of 763 cases. Heart, Lung and Circulation. 2008;17(6):502-4

5. McManus DP, Zhang W, Li J, Bartley PB. Echinococcosis. The Lancet. 2003;362(9392):1295-304.

6. Gottstein B, Reichen J. Hydatid lung disease (echinococcosis/hydatidosis). Clinics in chest medicine. 2002;23(2):397-408.

7. Junghanss T, Da Silva AM, Horton J, Chiodini PL, Brunetti E. Clinical management of cystic echinococcosis: state of the art, problems, and perspectives. The American journal of tropica medicine and hygiene. 2008;79(3):301-11.

8. Pallua K, Putz G, Mitterschiffthaler G, Brezinka C, Biebl M. Management of hepatic echinococcosis in pregnancy. International journal of gynaecology and obstetrics. 2010;109(2). 